\title{
Análisis de la Acción Tutorial y su Incidencia en el Desarrollo Integral del Alumnado. El Caso de la Universidad de Castilla La Mancha, España
}

\author{
Santiago Alonso-García ${ }^{(1)}$, Antonio M. Rodríguez-García(2)* y Ma. Pilar Cáceres-Reche ${ }^{(2)}$ \\ (1) Facultad de Ciencias de la Educación, Dpto. de Didáctica y Organización Educativa, Univ. de Sevilla, \\ Pirotecnia S/N, 4103 Sevilla - España. (e-mail: sag@us.es) \\ (2) Facultad de Ciencias de la Educación, Dpto. de Didáctica y Organización Escolar, Univ. de Granada, \\ Campus de Cartuja S/N, 18071 Granada - España. (e-mail: arodrigu@ugr.es; caceres@ugr.es)
}

*Autor a quien debe ser dirigida la correspondencia

Recibido Oct. 18, 2017; Aceptado Dic. 26, 2017; Versión final Ene. 25, 2018, Publicado Jun. 2018

\begin{abstract}
Resumen
Se presenta los resultados de un estudio en el que se analiza las percepciones de los estudiantes del Grado de Maestro de Educación Infantil y de Educación Primaria de la Facultad de Educación de la Universidad de Castilla La Mancha (España) acerca de cómo influye la tutoría universitaria en la mejora de su desarrollo integral. El desarrollo integral es analizado considerando tres dimensiones: personal, académico y profesional, tras la implantación del marco del Espacio Europeo de Educación Superior (EEES). Se ha seguido una metodología cuantitativa aplicando un cuestionario ad hoc a 493 estudiantes. Los resultados manifiestan la relevancia de la tutoría universitaria para dar respuesta a necesidades académicas, docentes, personales, profesionales y, en menor medida, burocráticos. Se observa también la influencia positiva de la tutoría para el seguimiento de las metodologías activas y como espacio de mejora de las relaciones entre profesores y alumnos.
\end{abstract}

\section{Analysis of the Tutorial Action and its Impact on the Overall Development of the Students. The Case of the University of Castilla La Mancha, Spain}

\begin{abstract}
The results of a study aimed to analyze the perception of university students of the degrees of Early Childhood Education and Primary Education of the University of Castilla La Mancha (Spain), on how university tutoring affects the improvement of the integral development of students. The integral development includes three dimensions: personal, academic and professional, after the implementation of the framework of the European Higher Education Area (EHEA). A quantitative study was carried out by applying a questionnaire to 493 students. The results show the usefulness of university tutoring to respond to academic, teaching, personal, professional and, to a lesser extent, bureaucratic needs. The usefulness of tutoring for the monitoring of active methodologies and as a space for improving relations between teachers and students is also observed.
\end{abstract}

Keywords: orientation; tutorial action; higher education; university; teacher training 


\section{INTRODUCCIÓN}

Hace prácticamente dos décadas del inicio de la creación e implantación del Espacio Europeo de Educación Superior (EEES). Varios aspectos se encontraron en el punto de mira de las reformas para ser tratados en las diferentes reuniones ministeriales realizadas en distintos enclaves del panorama europeo: nueva estructuración de titulaciones, revisión de metodologías docentes, actualización de contenidos, enfoques de enseñanza-aprendizaje centrados en el estudiante, nueva delimitación curricular propuesta por competencias, entre otras. La tutoría universitaria, por su parte, también forma parte de ese proceso de reestructuración de la educación superior para potenciar la acción docente en el que éste ha de encargarse de diseñar situaciones significativas de aprendizaje a la par que se ocupe de los aspectos de orientación personal, académica y profesional de sus alumnos (De Backer et al., 2015; Hagenauer y Volet, 2014; López-Gómez, 2017).

Históricamente, la tutoría, acción tutorial o proceso de orientación ha ido avanzando desde sus inicios basados en el diagnóstico como una acción puntual y sus diferentes enfoques (psicométrico, clínico-médico, humanista, sociológico y didáctico) hasta lograr una visión más amplia y comprensiva, teniendo en cuenta las diferentes etapas y necesidades de la persona (Amor-Almedina y Dios-Sánchez, 2017; Gaughf y Foster, 2016; O'Kelly et al., 2015). De este modo, avanzamos de una orientación reparadora a una orientación preventiva, sistemática, general y continuada destinada a promover el desarrollo integral de las personas y que debe estar presente en toda la vida del individuo (Gaughf y Foster, 2016; Sola y López, 2008). Se trata de un proceso que debe asentarse en tres principios básicos (Amor-Almedina, 2016; Martínez et al., 2014; Sola y López, 2008): 1) Principio de prevención: anticipación a las situaciones que van abordando al individuo a lo largo de su existencia, englobando factores como las distintas etapas educativas, la evaluación inicial y agentes externos; 2) Principio de desarrollo del individuo: provisión de personas con competencias adquiridas y necesarias para dar respuesta a las situaciones que le demanda la vida, sirviendo la orientación para facilitar el desarrollo personal, el auto concepto, la clarificación de valores y el desarrollo para la toma de decisiones; y 3) Principio de intervención educativa: y, a su vez, orientadora, toma en consideración no solamente al sujeto sino a las circunstancias que le rodean y en las que se desenvuelve.

Extrapolándolo al contexto de la academia, la acción tutorial en la universidad puede definirse, según Sanz (2009), como "una acción docente de orientación [...] con la finalidad de participar en la formación integral del alumnado potenciando su desarrollo como persona y como estudiante y su proyección social y profesional mediante la realización de estímulos que favorecen la reflexión crítica, la autocomprensión, la autoestima, la adaptación social, los recursos y la mejora del clima de y para el aprendizaje" (p.19).

Tras las orientaciones del Informe Universidad 2000 la tutoría universitaria debía transformarse en un espacio donde el docente ha de realizar tareas de asesoramiento a sus estudiantes abarcando diferentes procesos y situaciones: acceso previo al ingreso en la universidad; preparación y desarrollo de las habilidades educativas necesarias a desarrollar; planificación de los estudios; apoyos necesarios en casos de crisis, rencillas o dificultades particulares de algunos alumnos; asesoramiento al desarrollo y madurez formativa de los estudiantes; acompañamiento y orientación profesional, etc (Amor-Almedina y Dios-Sánchez, 2017; Mouraz y Sousa, 2016; Tralongo, 2017). El docente se convierte, pues, en un guía que ayuda en la toma de decisiones de los estudiantes, examinando diferentes alternativas y analizando las distintas consecuencias que pueden tener las mismas. En este sentido, según Arco et al. (2009), y siguiendo las directrices europeas, la acción tutorial en la universidad debe suplir cinco niveles de atención para contribuir al desarrollo personal, académico y profesional de los estudiantes: contenidos burocráticos (información al estudiante sobre los diferentes servicios de orientación académica, profesional y personal de la Universidad y de sus centros; información sobre diferentes tareas administrativas y burocráticas, etc.), académicos (información sobre la asignatura, resolución de dudas, seguimiento de trabajos y prácticas, revisión de la evaluación, mejora del aprendizaje, resolución de problemas, toma de decisiones, etc.), docentes ( planificación y organización de la materia, metodologías,...), personales (motivar al alumno, contribuir a la construcción de su autoconcepto, autoestima, habilidades sociales, resolución de problemas personales o de ajuste académico y social en el plano intelectual, social, emocional, institucional...) y profesionales (transición al mundo laboral, información sobre el mercado de trabajo, aprendizaje a lo largo de la vida, etc.). A tal efecto, López-Gómez (2017) señalaba que la tutoría universitaria "se configura como un proceso inherente a la función docente, desde la que se proyectan las nuevas expectativas y pretensiones que han sido directrices del EEES" (p.63). De esta manera, en la acción tutorial actual se apuesta por el desarrollo de diferentes enfoques de enseñanza que se centren en el estudiante, un proceso de mayor individualización de la formación, la apuesta por los modelos educativos integrales, el desarrollo de competencias, la orientación para el desarrollo profesional, etc.

Consecuentemente, en los últimos años hemos experimentado un crecimiento progresivo de servicios destinados a la orientación de alumnos en las diferentes universidades españolas (Amor-Almedina, 2016) y que vienen a suplir todas sus necesidades como estudiantes. Sin embargo, la tradición nos advierte que, en el caso de las tutorías docentes, aquello que realmente predomina en estos espacios que complementan su 
acción están dedicados casi en su globalidad a la resolución de dudas, revisión de exámenes, trabajos u otros aspectos relacionados con la materia que imparten (Amor- Almedina y Dios-Sánchez, 2017; Sanz, 2009). En este sentido, el estudiante universitario puede poseer tantos tutores como asignaturas o módulos esté cursando. Sin embargo, la tutoría no ha de concebirse como una función complementaria a la docencia, sino como un todo que engloba la acción realizada por el tutor, por lo que la docencia entra a formar parte de la tutoría junto con el resto de ámbitos-contenidos tutoriales (burocráticos, académicos, personales y profesionales) para dar respuesta a las necesidades que van surgiendo a lo largo de la trayectoria académica del estudiante.

En este contexto, los diferentes países de la Comunidad Europea (CE) han adoptado posiciones y sistemas distintos para dar respuesta a las necesidades de orientación de sus propios alumnos. Así, encontramos ejemplos como las universidades de Reino Unido, donde se apuesta por el desarrollo personal de sus estudiantes; instituciones de educación superior de Francia, que hacen énfasis en el desarrollo profesional de sus alumnos; mientras que las organizaciones alemanas de esta índole han hecho mayor hincapié en el valor de la investigación, el conocimiento y la trasferencia (Ceballos, 2017; López-Gómez, 2017; Tralongo, 2017; Winder et al., 2016). De este modo, aunque la tradición haya mostrado diferentes acciones llevadas a cabo en la tutoría universitaria, en función del contexto donde se inserta, la realidad actual tras la convergencia europea apuesta por un empoderamiento de la tutoría en la universidad (Cremades et al., 2016; De Backer et al., 2015; O'Kelly et al., 2015).

Adentrándonos en el contexto de esta investigación, la Universidad de Castilla la Mancha (UCLM) (España) no cuenta con un servicio de orientación unificado en su estructura, sino que su oferta se determina en la existencia de diferentes servicios de atención al alumnado de esta institución, donde encontramos: el Servicio de Atención Psicológica (SAP), el Servicio de Apoyo al Estudiante con Discapacidad (SAED) y el Centro de Información y Promoción del Empleo (CIPE). A nivel particular, la Facultad de Educación del Campus de Albacete (Castilla La Mancha, España) no dispone de ningún servicio específico de atención y orientación, por lo que toda esta labor recae en el buen hacer del equipo directivo, del personal docente e investigador y del personal de administración y servicios (Alonso, 2012). En este particular contexto, algunas herramientas sirven como instrumentos principales para llevar a cabo las tareas de orientación dentro de la Facultad mencionada, estando disponible un espacio web dentro del servidor de la universidad y llevando a cabo otras tareas específicas, donde cabe mencionar las jornadas de recepción que se realizan todos los años a los alumnos de primer curso, así como las posteriores jornadas referentes a los Prácticum I y II.

\section{METODOLOGÍA}

La presente investigación se encuadra dentro de una metodología de naturaleza cuantitativa, de carácter no experimental y trasversal con una idiosincrasia descriptiva a fin de analizar las percepciones de los estudiantes del Grado de Maestro de Educación Infantil y de Educación Primaria de la Facultad de Educación de la Universidad de Castilla La Mancha (Campus de Albacete) (España) acerca del impacto de la acción tutorial en el desarrollo integral del alumnado integrado por tres dimensiones: personal, académico y profesional, tras la implantación del marco del Espacio Europeo de Educación Superior (EEES).

\section{Objetivos específicos}

En línea con el anterior objetivo general, nuestro trabajo contempla dos objetivos específicos: 1) Conocer la influencia de la tutoría universitaria ejercida por los docentes para dar respuesta a contenidos burocráticos, académicos, docentes, personales y profesionales de los estudiantes; y 2) Determinar la utilidad de la tutoría universitaria para trabajar en línea de las directrices que marca el EEES para mejorar la acción tutorial en el contexto de la academia.

\section{Participantes y contexto}

La presente investigación se desarrolla Facultad de Educación de la Universidad de Castilla La Mancha (Campus de Albacete) durante el curso 2015-2016. Una vez se realizaron las pertinentes diligencias con la institución para la obtención del tamaño total de nuestro universo de estudio, obtuvimos que la totalidad de la población se corresponde a N=1224 sujetos, siendo 794 quienes cursan el Grado de Maestro en Educación Primaria y 430 quienes se encuentran matriculados en el Grado de Maestro de Educación Infantil.

En instas de obtener el mayor índice de respuesta posible por parte de la población de estudio, optamos por invitar a la globalidad de los estudiantes para ser partícipes en el mismo. En este sentido, de los 1224 alumnos que forman parte de la población, 493 complementaron de manera satisfactoria el instrumento administrado. De este modo, contamos con un índice de respuesta del $40,27 \%$ superando, por tanto, el $30 \%$ de respuesta necesario para la representatividad de estudios por encuesta (Rodríguez y Valldeoriola, 2009). 
Procediendo a desglosar detalladamente los datos de la muestra de la investigación, 259 alumnos (52,5\%) se encuentran matriculados en el Grado de Maestro de Educación Primaria, mientras que los 234 estudiantes restantes (47,5\%) cursan los estudios para la obtención del título del Grado de Maestro de Educación Infantil. Atendiendo a los datos demográficos de la muestra participante, el $18 \%$ son hombres mientras que el $82 \%$ restante son mujeres, con edades que van entre los 18 hasta superar los 40 años de edad (tabla 1).

Tabla 1: Estadísticos de Edad

\begin{tabular}{|lr|r|r|}
\hline & & Frecuencia & Porcentaje \\
\hline Válidos & $18-25$ & 428 & 86,8 \\
\cline { 3 - 4 } & $26-30$ & 44 & 9,0 \\
\cline { 3 - 3 } & $31-35$ & 10 & 2,0 \\
\cline { 3 - 3 } & $36-40$ & 5 & 1,0 \\
\cline { 3 - 3 } & $>40$ & 4 &, 8 \\
\cline { 3 - 3 } & Total & 491 & 100,0 \\
\cline { 3 - 3 } Perdidos & NS/NC & 2 & \\
\cline { 3 - 3 } Total & & 493 & \\
\cline { 3 - 3 } & &
\end{tabular}

\section{Instrumento}

Respecto al instrumento empleado para la recogida de datos, se procedió al diseño e implementación de un cuestionario construido ad hoc (Alonso, 2012). El instrumento estaba compuesto por un total de 135 ítems quedando posteriormente reducido a 65 tras los procesos de validación, y donde se abordó ampliamente la temática del estudio, así como aspectos sociodemográficos inherentes a cualquier investigación en ciencias sociales (edad, sexo, nivel de estudios alcanzado, etc.). Se trata de una modalidad de cuestionario Likert, siendo cada enunciado presentado en una escala de estimación con cuatro alternativas de respuesta: 1 (totalmente en desacuerdo), 2 (en desacuerdo), 3 (de acuerdo) y 4 (totalmente de acuerdo). Tras la validación de expertos, los índices de fiabilidad calculados a través del alfa de Cronbach, obtuvieron un valor de 0,967 para 65 elementos; valor superior al establecido por la comunidad científica para garantizar la consistencia del instrumento (Rodríguez y Valldeoriola, 2009).

\section{Análisis de datos}

Para dar respuesta a los objetivos planteados en esta investigación se ha efectuado un tratamiento y posterior análisis de los datos a través del paquete estadístico SPSS en su versión 23.0. Para ello, se ha hecho uso principalmente de los parámetros de la estadística descriptiva, valiéndonos principalmente del empleo de porcentajes y frecuencias en instas de promover una visión general del problema que aquí abordamos.

\section{RESULTADOS}

Para conocer las percepciones del estudiante universitario acerca de la impacto de la tutoría universitaria ejercida por el cuerpo de profesores se realizaron diferentes cuestiones a los alumnos de estas titulaciones referentes a la atención recibida e información ofertada durante el desarrollo de la acción tutorial y la percepción de utilidad de las mismas para responder a sus necesidades como estudiantes en diferentes ámbitos: burocrático (e.g.: información sobre los diferentes servicios de orientación académica, profesional y personal de la Universidad y de sus centros; información sobre diferentes tareas administrativas y burocráticas, etc.), académico (e.g.: información sobre la asignatura, resolución de dudas, seguimiento de trabajos y prácticas, revisión de la evaluación, mejora del aprendizaje, resolución de problemas, toma de decisiones, etc.), docente (e.g.: planificación y organización de la materia, metodología docente para profundizar en los contenidos abordados en clase), personal (e.g.: motivación, autoconcepto, autoestima, habilidades sociales, integración en un grupo, participación en contextos comunitarios, resolución de problemas personales o de ajuste académico y social en el plano intelectual, social, emocional, institucional...) y profesionales (e.g.: transición al mundo laboral, itinerario curricular, elección de un proyecto profesional, información sobre el mercado de trabajo, salidas profesionales, aprendizaje a lo largo de la vida, etc.) (Fig.1).

Si observamos la gráfica, con un primer vistazo resulta fácil deducir que la mayoría de los alumnos se encuentran de acuerdo (49,7\%) respecto a la utilidad de la tutoría para esclarecer aspectos del ámbito académico, seguida de cerca por aquellos que valoran el provecho de este espacio para ayudar en cuestiones relacionadas con aspectos docentes (48,3\%), personales (40,7\%), profesionales $(40,2 \%)$ y, en último lugar, se encontrarían las acciones encaminadas a responder las necesidades de índole burocrático (29,5\%). En este último ítem al que hacemos referencia, se han encontrado valores más dispares en comparación con aquellos otros analizados de su misma categoría, manifestando un vacío existente respecto a las necesidades que presentan los alumnos para dar respuesta a sus necesidades burocráticas en el transcurso de sus estudios. 


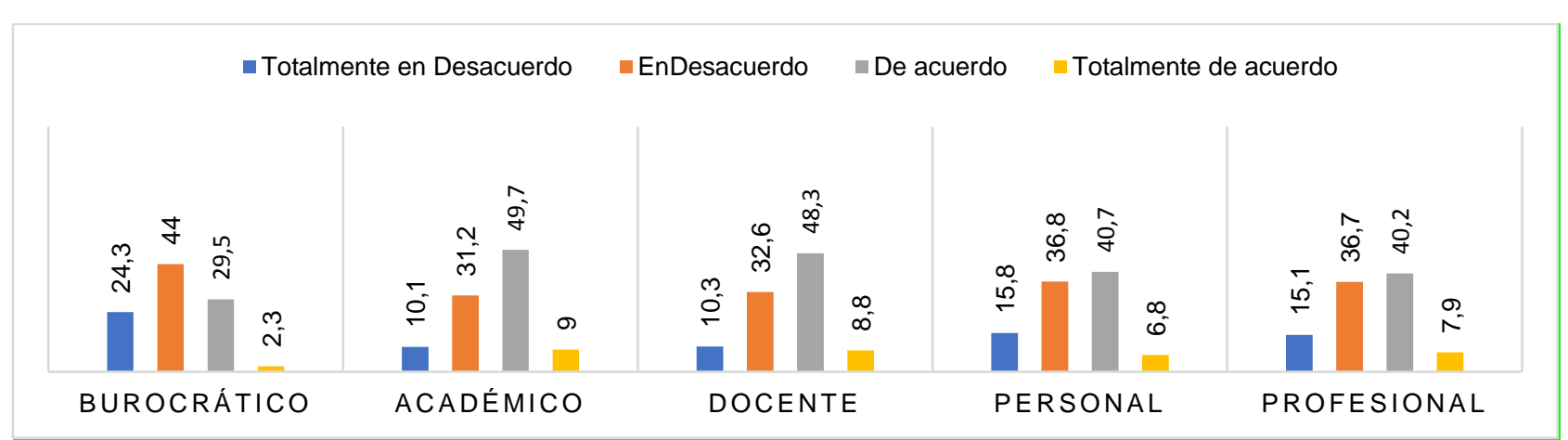

Fig. 1: Valoración de la utilidad de la orientación en función del ámbito

Si analizamos de una forma más detallada cada una de las dimensiones representadas la gráfica anterior, en lo que respecta a la tutoría como acción de utilidad para desarrollar y atender los contenidos burocráticos (CB) obtiene una media de respuesta de 2,67 y una desviación típica de 0,839. Se observa que la respuesta que está secundada por un mayor número de sujetos (44\%) es precisamente la que está en desacuerdo con la utilidad de la información recibida para la resolución de sus necesidades de estudiante universitario en este aspecto, lo que revela que junto con la otra valoración negativa de totalmente en desacuerdo (24'3\%) la existencia de un grado de disconformidad bastante elevado. Por el contrario, solamente el $31,8 \%$ de la muestra valora positivamente este espacio para informar acerca de diferentes tareas administrativas y burocráticas que afectan al estudiante universitario, por ejemplo.

Por otro lado, respecto a la adecuación de los contenidos académicos de la acción tutorial (CAC), se ha obtenido una puntuación media de 2,58 y una desviación típica de 0,792. Al contrario que ocurre con el ítem anterior, se observa que la respuesta que está apoyada por un mayor número de sujetos (49'7\%), es precisamente la que está de acuerdo con esta afirmación, lo cual revela que, junto con la otra valoración positiva de totalmente de acuerdo $(9 \%)$, existe un alto porcentaje de alumnos que consideran de utilidad la información presentada a nivel académico para suplir sus necesidades de este tipo en el transcurso de su carrera. En contraposición a esta afirmación, encontraríamos a un $41,3 \%$ de los estudiantes que afirman estar en desacuerdo o totalmente en desacuerdo. De este modo, nos encontramos ante dos bloques de opiniones bien consolidados que se encuentran en lados opuestos, percibiendo que hay gran porcentaje de alumnos que no afirman recibir una información adecuada para suplir sus necesidades en esta dimensión.

La variable que se refiere a la utilidad y adecuación de los contenidos docentes de la acción tutorial (CDO) obtenemos una puntuación media respecto a la valoración con un valor de 2,56 y una desviación típica de 0,794 . Atendiendo al porcentaje de respuesta, entre el alumnado destaca una valoración positiva levemente superior sobre el resto, con un $57,1 \%$ (48,3\% de acuerdo y $8,8 \%$ totalmente de acuerdo), acerca de la utilidad de la acción tutorial universitaria para suplir los contenidos docentes, es decir, aquellos relacionados con la planificación y organización de la asignatura. No obstante, el $42 \%$ restante expresa su desacuerdo respecto a esta variable, por lo que existen una gran cantidad de alumnos que no se encuentran conformes con tales afirmaciones.

La dimensión que analiza la adecuación de los contenidos personales de la acción tutorial (CPE) obtiene una media de 2,38 y una desviación típica de 0,830 , siendo mayoritariamente aquellos que se tornan en una posición en desacuerdo o muy en desacuerdo $(52,6 \%)$ respecto a los que afirman lo contrario $(47,5 \%)$. En otras palabras, la mayoría de alumnos se encuentra disconforme con el programa desarrollado en la tutoría universitaria por el docente para contribuir al desarrollo de sus necesidades personales, relacionadas con el desarrollo de la motivación, la integración en un grupo, la autoestima, etc.

En último lugar, la variable que se refiere a la utilidad y adecuación de los contenidos profesionales de la acción tutorial (CPR) para suplir las necesidades de los estudiantes universitarios ha obtenido una puntuación media de 2,49 y una desviación típica de 0,831. En este sentido, la mayoría de estudiantes se torna en una posición desfavorable respecto a esta dimensión con un grado de desacuerdo levemente superior a la mitad de los encuestados $(51,8 \%)$, mientras que aquellos otros que afirman que la información recibida les ha sido de utilidad para suplir sus necesidades profesionales se han posicionado hacia este ítem con un $48,1 \%$ de acuerdo. De este modo, existe una mayor proporción de estudiantes que se encuentra disconforme con la información recibida para su desarrollo profesional.

Ahondando en la cuestión en instas de profundizar en la tutoría como acción de utilidad para trabajar en línea de las directrices que marca el EEES y mejorar la acción tutorial en este contexto, se procedió a preguntar la opinión de los alumnos en relación a la tutoría como acción de utilidad para potenciar el uso de las TIC y de 
Internet como medios de comunicación entre el docente y los alumnos, ya sea a través de redes sociales, mensajería en tiempo real, e-mail, vídeo tutoría, etc.; para el consenso del horario de la tutoría con el alumnado (grupo-clase); la resolución de posibles conflictos de intereses entre compañeros; el fomento de la empatía con los tutores y alumnos; la resolución de posibles conflictos entre tutor-alumno; así como la comprensión sobre los aspectos organizativos de la función tutorial como, por ejemplo, la asignación de espacios, modalidades, agrupamientos, temporización, entre otros (Figuras 2 y 3 ).

Haciendo una primera aproximación general de los datos que en estas figuras se recogen, observamos que los alumnos han percibido la tutoría universitaria como herramienta de mayor utilidad en el desarrollo de estrategias adicionales para la utilización, desarrollo y seguimiento de las metodologías activas promovidas por el EEES $(50,8 \%)$, así como para el acuerdo de citas y horario de atención del alumnado en beneficio mutuo $(49,8 \%)$, para la resolución de conflictos entre pares $(49,6 \%)$, entre profesores y alumnos $(46 \%)$, la clarificación sobre otros aspectos organizativos (45,4\%), las acciones para mejorar la empatía entre el docente y los alumnos $(43,2 \%)$ y, en último lugar, las acciones tutoriales realizadas para el empleo y fomento del uso de las TIC e Internet (36,4\%), siendo este último ítem el que ha recibido un menor grado de concentración de respuestas por parte de los alumnos.

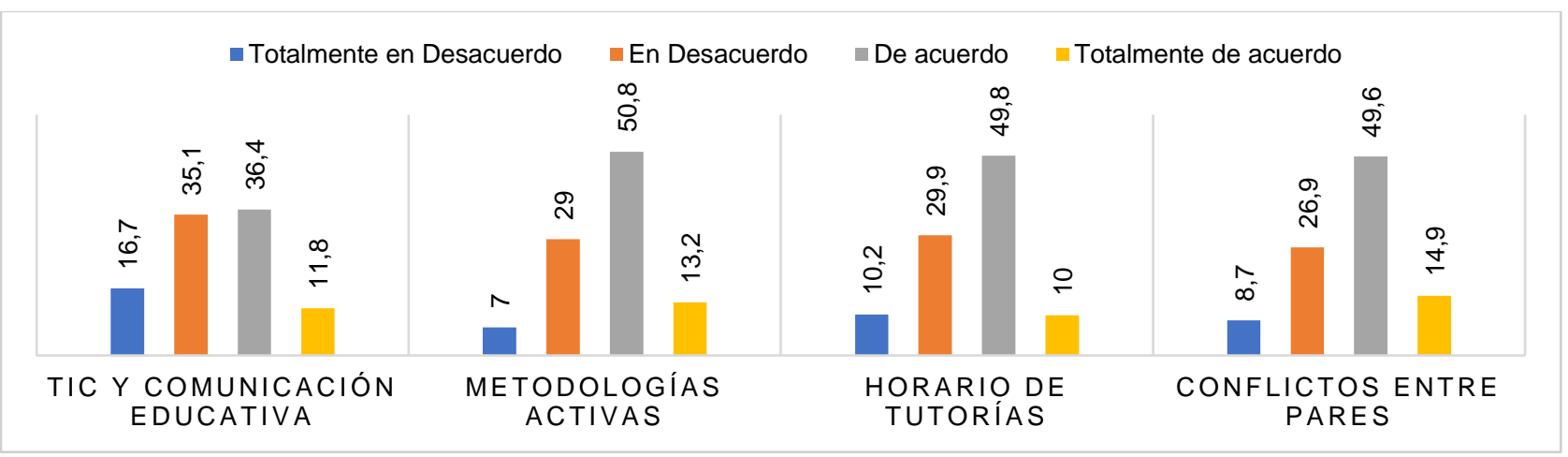

Fig. 2: Acciones de utilidad de la tutoría (I)

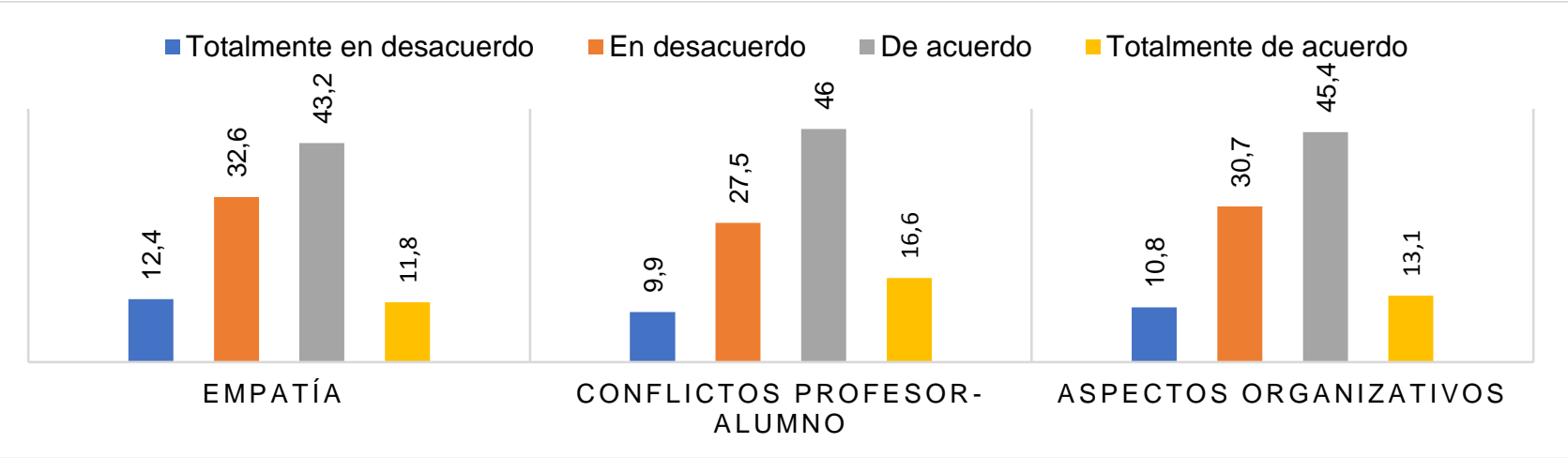

Fig. 3: Acciones de utilidad de la tutoría (II)

Si atendemos a los datos de manera pormenorizada, en relación al ítem que analiza la utilidad de la tutoría para promover el uso de las TIC e Internet como medios de comunicación educativa (UUI), encontramos una media con un valor de 2,43 y una desviación típica de 0,904 . Se observa que la respuesta que está secundada por un mayor número de alumnos (36'4\%), es precisamente la que está de acuerdo con esto. No obstante, son la mayoría de sujetos quienes se encuentran en una opinión desfavorable respecto a esta variable, con un total de $51,8 \%$ que se posicionan en desacuerdo o muy desacuerdo en la escala suministrada. En este sentido, los alumnos se han sentido disconformes con la acción tutorial universitaria como acción de utilidad para promover el uso de las TIC en el desarrollo de la tutoría (videollamadas, chats, redes sociales, etc.).

Haciendo alusión a la variable que analiza la tutoría como espacio de utilidad a la hora de continuar con el apoyo para trabajar diferentes metodologías activas de trabajo aplicables a diferentes situaciones de enseñanza-aprendizaje y dar respuesta a los cambios producidos por las mismas (UTM), se obtiene una puntuación media de 2,70 y una desviación típica de 0,784. Observamos, como hemos comentado anteriormente, un alto grado de acuerdo en esta pregunta, con un 64\% procedente de la suma de las dos respuestas afirmativas. Estos datos nos arrojan información importante acerca de la importancia de la tutoría para fomentar y apoyar el desarrollo de metodologías activas de enseñanza-aprendizaje en la universidad. 
Avanzando hacia la dimensión que analiza la tutoría como acción de utilidad para consensuar nuevas o futuras citas con el alumnado (UCH) se obtiene una puntuación media de 2,60 y una desviación típica de 0,805, siendo mayoritarios aquellos que se posicionan en una posición de acuerdo en este ítem con un $49,8 \%$ y un $10 \%$ respectivamente frente a aquellos que no coinciden con sus compañeros, en un $26,9 \%$ y un $10,2 \%$. En este sentido, la mayoría de los alumnos percibe la tutoría como un espacio útil de reunión para el concierto de nuevas citas de acuerdo a sus necesidades específicas como estudiante universitario.

Por otro lado, en relación a la cuestión que se encarga de analizar la tutoría como acción de utilidad para la resolución de posibles conflictos de intereses entre compañeros (URA), tal y como marcan las directrices del EEES, se obtiene una puntuación media de 2,71 y una desviación típica de 0,824. En esta dimensión, por tanto, la mayoría de alumnos confiere un gran nivel de utilidad a las acciones desempeñadas para solventar esta problemática con un $64,5 \%$ de grado de acuerdo, mientras que el $35,6 \%$ restante difiere de la opinión de sus compañeros. De este modo, se entiende que la tutoría se ha convertido en un espacio de utilidad para solventar problemas entre el grupo.

El análisis de la pregunta que investiga la tutoría como acción de utilidad para el fomento de la empatía de los alumnos con los tutores (UFE) ha obtenido una puntuación media de 2,54 y una desviación típica de 0,856. En esta variable observamos que las mayores concentraciones de respuestas se sitúan en torno a las posiciones favorables con un $55 \%$ del total, siendo el $45 \%$ restante quienes opinan lo contrario. Este ítem nos demuestra que gran parte de los alumnos se sitúan en una posición positiva respecto a la utilidad del espacio de tutoría para entablar conversaciones de índole más personal que lleguen a comprender dificultades en el proceso de enseñanza-aprendizaje.

Analizando la dimensión que indaga la acción de utilidad de la tutoría para la resolución de posibles conflictos entre profesores y alumnos (UCA), se ha obtenido una puntuación media de 2,69 y una desviación típica de 0,863. La mayor concentración de respuestas se sitúa en el lado positivo, secundado por aquellos alumnos que se posicionan favorablemente respecto a la utilidad de estos espacios para la mejora de las relaciones entre profesores y alumnos con un $62,6 \%$ que concentra las opiniones positivas. Al contrario, se encontrarían aquellos que difieren de sus compañeros con un 37,4\%. En otras palabras, la mayor parte de los alumnos define la tutoría universitaria como espacio de utilidad para la resolución de conflictos entre alumnos y profesores.

En último lugar, el ítem que examina la tutoría como una acción de utilidad para la gestión de otros aspectos organizativos de la acción tutorial (espacios, modalidades, agrupamientos, temporización, entre otros) (UAO) obtiene una puntuación media de 2,61 y una desviación típica de 0,804. En este enunciado, son la mayoría de alumnos (58,5\%) quienes se sitúan en una posición favorable con su opinión respecto a la utilidad de la tutoría para cuestiones organizativas, siendo menor el porcentaje de alumnos que opinan lo contrario $(41,5 \%)$. Estos datos nos informan que los alumnos perciben la acción tutorial como un espacio de utilidad para gestionar otros aspectos organizativos que afectan al desarrollo del estudiante universitario.

\section{DISCUSIÓN}

La transformación del rol docente como persona que ha de encargarse de diseñar situaciones significativas de aprendizaje y, a la vez, debe ocuparse de los aspectos de orientación personal, académica y profesional de sus estudiantes definen la esencia de esa metamorfosis que requirió el cambio de rol de éste tras el proceso de convergencia. Las exigencias europeas requerían de la creación de un espacio de tutoría universitaria en la que el docente pudiese dar respuesta a diferentes contenidos y ámbitos que contempla la tutoría universitaria (aspectos de información sobre los diferentes servicios de orientación, tareas administrativas, seguimiento de la asignatura, generar un clima efectivo en las relaciones con su alumnado, fomento de la empatía, mejora de la motivación de los alumnos, su autoestima, brindarle oportunidades e información para su desarrollo profesional, entre muchas otras (Lee et al., 2017).

Siguiendo las cinco dimensiones propuestas por Arco et al. (2009) para responder a todas las necesidades y peculiaridades del estudiante a lo largo de su transcurso como universitario siguiendo lo establecido por Europa. En este sentido, a raíz de los resultados encontrados, los estudiantes perciben que la tutoría universitaria en el contexto de la investigación ha sido de mayor utilidad para el desarrollo de contenidos académicos, docentes, personales y profesionales. En este sentido, la suma de todos ellos, junto a los burocráticos, contribuyen al desarrollo personal, académico y profesional del individuo en su etapa universitaria, tal y como señalan Da Re et al., (2017) en su investigación acerca de la implantación del modelo de tutoría universitaria española en una universidad italiana.

En línea del párrafo anterior, destaca la tutoría como espacio para orientar el desarrollo personal del individuo, en el que se establecen canales bidireccionales de conversación que trabajan y mejoran la motivación del 
estudiante, su autoconcepto, autoestima, habilidades sociales, integración en el grupo, etc.), tal y como muestra la investigación de Lee et al., (2017). En lo que respecta a la orientación y desarrollo profesional del alumno universitario, los estudiantes perciben la acción tutorial como un proceso de utilidad para la obtención de información acerca de las diferentes posibilidades que disfrutan para mejorar su transición hacia el mundo laboral, información sobre el mercado de trabajo, salidas profesionales, entre otros, aspectos a tener muy presentes para la implementación de mejoras en la tutoría académica como acción de calidad (Dropicio, 2013). En último lugar, se encontrarían las acciones tutoriales que han servido para ayudar en aspectos burocráticos como, por ejemplo, información sobre los servicios de orientación académica, personal y profesional de la universidad y sus centros, información sobre tareas administrativas y burocráticas, entre otros, como ya han manifestado otras investigaciones (Martínez, Martínez y Pérez, 2014). Todo ello nos revela la necesidad de seguir trabajando en la calidad de los procesos tutoriales en el ámbito universitario, aspecto muy necesario para el desarrollo de la persona como ser integral.

Sin embargo, aunque a priori parecen haberse obtenido resultados eminentemente positivos, se percibe un gran sector de estudiantes que manifiestan la escasa utilidad de la acción tutorial para suplir sus necesidades como estudiantes, especialmente en el ámbito personal $(52,6 \%)$, profesional $(51,8 \%)$ y burocrático $(68,3 \%)$. Atendiendo a este dato, deducimos que los estudiantes siguen asociando en mayor medida la tutoría universitaria como una actividad que se reduce a la resolución de dudas, revisión de exámenes, entrega de trabajos, planificación y organización de la asignatura, resolución de conflictos, seguimiento de la asignatura, entre otros. Resultados similares pueden observarse en las investigaciones de Aguaded y Monescillo (2013), Aguilar-Salinas, Chávez-Valenzuela y de las Fuentes-Lara (2017), Amor-Almedina y Dios-Sánchez (2017) y Sanz (2009).En este sentido, pese a los cambios inroducidos para las reformas de la educación superior a nivel europeo, todavía se siguen manteniendo ciertas tendencias de la tutoría universitaria como espacio de utilidad mayormente destinado a aspectos relacionados con la materia que están cursando.

Por otro lado, el proceso de tutoría no debe concebirse como una mera orientación académica, sino que también debe englobarse bajo la dimensión humana y social que tiene la universidad. Así pues, el personal de estas instituciones no solamente se enfrenta al desarrollo personal, académico y profesional de los futuros estudiantes, sino también a hacer dicho proceso acorde a las directrices del EEES; esto es, incluyendo metodologías activas, programas de atención tutorial presenciales y virtuales más adecuados y útiles con los tiempos actuales, así como el desarrollo de competencias y habilidades generales y específicas de cada titulación, la convergencia hacia nuevos modelos educativos liberadores, la resolución de conflictos y prevención del abandono temprano, entre otros (Amor-Almedina y Dios-Sánchez, 2017; Hinojo, Aznar y Cáceres, 2009; Quoß, Rüttermann, y Gerhardt-Szep, 2017). En este sentido, los estudiantes universitarios participantes en esta investigación han percibido la tutoría universitaria como útil para el seguimiento de las metodologías activas de enseñanza-aprendizaje; como espacio para dialogar acerca de diferentes necesidades, para la resolución de conflictos, mejora de la relación entre alumnos y profesores, así como para gestionar otros aspectos organizativos que afectan al desarrollo del estudiante de universidad. Sin embargo, destaca un déficit en lo que respecta a la promoción del uso de las TIC para el desarrollo de la tutoría. Por ello, es preciso hacer hincapié en el desarrollo de diferentes medios de atención al estudiante, siendo la tutoría online uno de los más idóneos para desarrollar un mayor compromiso del estudiante con su aprendizaje (ChanLin, 2016).

Asimismo, la tutoría universitaria debe contribuir a integrar al estudiante y mejorar su proceso de adaptación en el sistema universitario, así como a apoyar e impulsar el desarrollo de su carrera, la transición posterior al mundo laboral y a la apuesta por la formación continua. Además, debe ayudar a satisfacer cualquier particularidad que emane de las nuevas necesidades producidas por los cambios políticos, sociales y económicos actuales (Ceballos, 2017). En este sentido, la acción tutorial dentro de la universidad facilita a los estudiantes la consecución de esa serie de habilidades, actitudes y aptitudes que son necesarias para su desarrollo personal y profesional en la universidad y en etapas posteriores et al., 2017; López-Gómez, 2017; Galbraith y Winterbottom, 2011; Loke y Chow, 2007).

\section{CONCLUSIONES}

A modo de síntesis, la acción tutorial en la universidad puede concebirse como un conjunto de procesos encaminados a la adquisición de competencias vitales por parte del alumnado, tales como: aprender a aprender, aprender a vivir y aprender a trabajar (Amor-Almedina, 2016; Sanz, 2009). Más concretamente, Sanz (2009) realiza especial mención al espacio de tutoría como elemento para favorecer el desarrollo académico del alumno o el aprender a aprender, que se englobaría dentro de las acciones de mejora de sus aprendizajes, resolución de problemas y toma de decisiones. Por otro lado, respecto a su desarrollo personal y social o aprender a ser/estar contemplaría acciones como la mejora de la motivación, el autoconcepto, la comprensión, la empatía, autoestima, interacción en un grupo, etc. 
Finalmente, el desarrollo de la carrera o el aprender a hacer/trabajar comprendería todos aquellos aspectos relacionados con la obtención de información, el aprendizaje a lo largo de la vida, la toma de conciencia de su itinerario curricular, el acceso a la profesión, etc. En esta línea, es innegable el salto que supone pasar de un instituto de educación secundaria a un centro universitario, así como todos los cambios personales, psicológicos y sociales que ello supone. Por ello, es fundamental que este proceso sea abordado y ofrecido como un servicio de gran calidad (Aguaded y Monescillo, 2013; Arco et al, 2009; Casado et al, 2014; Dropicio, 2013; Martínez, Martínez y Pérez, 2014; Narro y Arredondo, 2013).

\section{REFERENCIAS}

Aguaded, C. y M. Monescillo, Evaluación de la tutoría en la Universidad de Huelva desde la perspectiva del alumnado de psicopedagogía: propuestas de mejora, Tendencias pedagógicas: (21), 163-176 (2013)

Aguilar-Salinas, W.E., Chávez-Valenzuela, G. E. y M. de las Fuentes-Lara, Tutorías: Estudio Exploratorio sobre la Opinión de los Estudiantes de Tronco Común de Ciencias de la Ingeniería, Formación Universitaria: 10(3), 69-80 (2017)

Alonso, S., Percepciones de la función tutorial como competencia básica del docente en el Espacio Europeo de Educación Superior (EEES): la Universidad de Castilla-La Mancha, Tesis de Magister, Dpto. Didáctica y Organización Escolar, Universidad de Castilla-La Mancha, España (2012)

Amor-Almedina, M. I. e I. Dios-Sánchez, La tutoría universitaria: un espacio para la orientación personal, académica y profesional en la formación inicial del profesorado, Rev. Española de Orientación y Psicopedagogía: 28(1), 119-130 (2017)

Amor-Almedina, M. I., Evaluación de la orientación y la tutoría en la Facultad de ciencias de la Educación de la Universidad de Córdoba, Educatio Siglo XXI: 34(2), 93-112 (2016)

Arco, J. L., López-Ortega, S., Fernández, F.D., Giménez, E., Caballero, R. y V. Heilborn, Guía Psicopedagógica para estudiantes y profesores universitarios en el Espacio Europeo de Educación Superior, $1^{\text {a }}$ edición, 1-193, Universidad de Granada, Granada, España (2009)

Casado, R., Greca, I.M., Trico, V., Collado, M. y A.M. Lara, Impacto de un plan de acción tutorial universitario: resultados académicos, implicación y satisfacción, Revista de Docencia Universitaria: 12(4), 86-103 (2014)

Ceballos, N., La tutoría como espacio de democracia: una mirada desde la voz de los docentes en formación, Revista Española de Orientación y Psicopedagogía: 28(1), 86-103 (2017)

ChanLin, L. J., Students' Involvement and Communit Support for Service Engagement in Online Tutoring, Journal of Educational Media y Library Sciences: 53(2), 245-268 (2016)

Chois-Lenis, P. M., Casas-Bustillo, A. C., Lopez-Higuera, A., Prado-Mosquera, D. M., y E. Cajas-Paz, E., Perceptions of Peer Tutoring in Academic Writing, MAGIS-Revista Internacional de Investigacion En Educacion: 9(19), 165-184 (2017)

Cremades Andreu, R., García Gil, D., Ramírez Rico, E. y E. Miraflores Gómez, Acción tutorial en estudiantes de las menciones de educación física música del grado de maestro en educación primaria, Revista de Investigación Educativa: 34(2), 417 (2016)

Da Re, L., Clerici, R., y P.R. Álvarez Pérez, The formative tutoring programme in preventing universit drop-outs and improving students' academic performance. The case stud of the Universit of Padova, Italian Journal of Soociology of Education: 9(3), 156-175 (2017)

De Backer, L., Van Keer, H. y M. Valcke, Promoting universit students' metacognitive regulation through peer learning: the potential of reciprocal peer tutoring, Higher Education: 70(3), 469-486 (2015)

Dropicio, E., Tutoría universitaria: propuestas didácticas de competencia tutorial, Revista de Docencia Universitaria, 11(2), 195-220 (2013)

Galbraith, J., y M. Winterbottom, M., Peer-tutoring: what's in it for the tutor?, Educational Studies:37(3), 321-332 (2011)

Gaughf, N., y P. Foster, Implementing a centralized institutional peer tutoring program, Education for Health: 29(2), 148 (2016)

Hagenauer, G., y S.E. Volet, Teacher-student relationship at universit: an important et under-researched field: Oxford Review of Education, 40(3), 370-388 (2014)

Hinojo, F.J., Aznar, I., y M. P. Cáceres, Percepciones del alumnado sobre el blended learning em la Universidad, Comunicar: 33, 165-174 (2009)

Lee, H.-J., Hong, y H. Choi, Perceptions of tutoring roles and pschological distance among instructors, tutors and students at a Korean universit, Higher Education Research y Development: 36(1), 143-157 (2017).

Loke, A. J. T. ., y F. L. W. Chow, Learning partnership-the experience of peer tutoring among nursing students: A qualitative study, International Journal of Nursing Studies: 44(2), 237-244 (2007)

López-Gómez, E., El concepto y las finalidades de la tutoría universitaria: una consulta a expertos, Revista Española de Orientación Psicopedagogía: 28(2), 61-78 (2017)

Martínez, P., Martínez, M., y J. Pérez, Tutoría Universitaria: entorno emergente en la Universidad Europea. Un estudio en la Facultad de Educación de la Universidad de Murcia, Revista de investigación educativa: 32(1), 111-138 (2014) 
Mouraz, A., y A. Sousa, An Institutional Approach to First-ear Adjustment. Journal of Hispanic Higher Education: 15(3), 221-239 (2016)

Narro, J., y M. Arredondo, La tutoría: un proceso fundamental en la formación de los estudiantes universitarios, Perfiles educativos: 35(141), 132-151 (2013)

O'Kell, M., Garrison, J., Merr, B., y J. Torreano, Building a Peer-Learning Service for Students in an Academic Librar. Portal, Libraries and the Academ: 15(1), 163-182 (2015)

Quoß, M., Rüttermann, S., y S. Gerhardt-Szep, Cross-ear peer-assisted learning using the inverted ("flipped") classroom design: A pilot stud in dentistry, Zeitschrift Für Evidenz, Fortbildung Und Qualität Im Gesundheitswesen: 126, 84-93 (2017)

Rodríguez, D. y J. Valldeoriola, Metodología de la Investigación. Barcelona: F.U.O.C (2009)

Sanz, R., Tutoría y atención personal al estudiante en la Universidad. Madrid: Síntesis (2009)

Sola, T. y N. López, Bases teóricas para humanizar la educación a través de la orientación la acción tutorial. Madrid: Universitas (2008)

Tralongo, S. Fabriquer / devenir un enseignant du supérieur professionnalisant, Le tutorat en IUT: 138(2), 165-183 (2017).

Winder, R., Kathpalia, S. S., y S. L. Koo, Writing centre tutoring sessions: addressing students' concerns, doi:10.1080/03055698.2016.1193476, Educational studies: 42(4), 323-339 (2016) 